TecnoLógicas

ISSN-p 0123-7799

ISSN-e 2256-5337

Vol. 22, No. 46, pp 213-231

Sep-dic de 2019

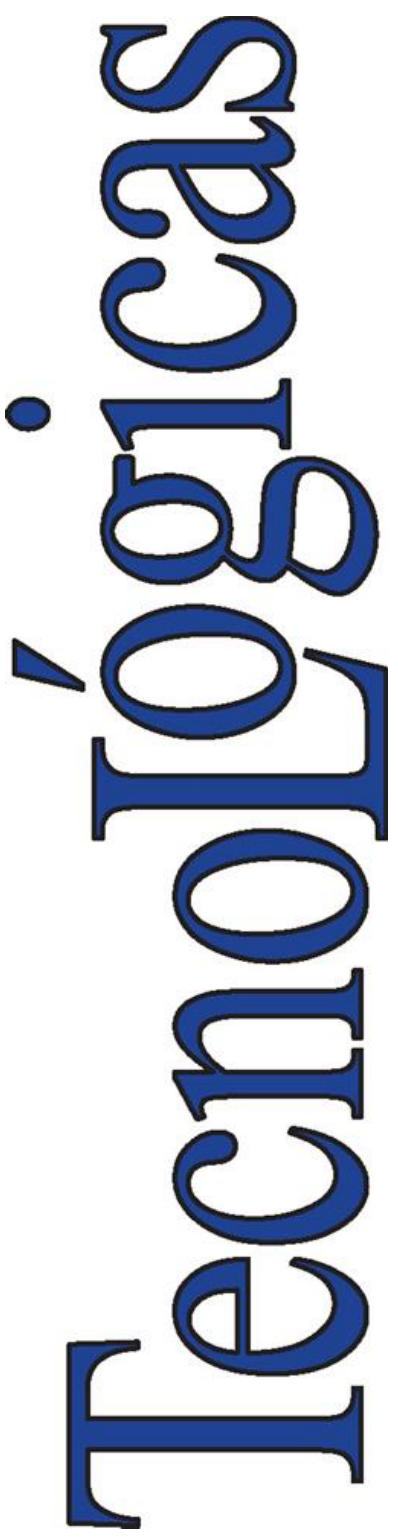

(C) Instituto Tecnológico Metropolitano Este trabajo está licenciado bajo una Licencia Internacional Creative Commons Atribución (CC BY-NC-SA)

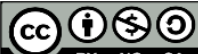

Artículo de Investigación/Research Article

\section{Evaluación del aporte de la covarianza de las señales electroencefalográficas a las interfaces cerebro-computador de imaginación motora para pacientes con lesiones de médula espinal}

\section{Assessing the Contribution of Covariance Information to the Electroencephalographic Signals of Brain-Computer Interfaces for Spinal Cord Injury Patients}

\author{
Carlos Ferrin-Bolaños (D) \\ Humberto Loaiza-Correa (D), \\ Jean Pierre-Díaz iD 3 , Paulo Vélez-Ángel(D4
}

Recibido: 05 de marzo de 2019

Aceptado: 19 de junio de 2019
Cómo citar / How to cite

C. Ferrin-Bolaños, H. Loaiza-Correa, J. Pierre-Díaz, P. Vélez-Ángel, "Evaluación del aporte de la covarianza de las señales electroencefalográficas a las interfaces cerebro-computador de imaginación motora para pacientes con lesiones de médula espinal". TecnoLógicas, vol. 22, no. 46, pp. 213-231, 2019.

https://doi.org/10.22430/22565337.1392

1 M.Sc. en Ingeniería Electrónica, Grupo de Percepción y Sistemas Inteligentes, Facultad de Ingeniería, Universidad del Valle, Cali-Colombia, carlos.ferrin@correounivalle.edu.co

2 PhD. en Robótica, Grupo de Percepción y Sistemas Inteligentes, Facultad de Ingeniería, Universidad del Valle, Cali-Colombia, humberto.loaiza@correounivalle.edu.co

3 Ing. Electrónico, Grupo de Percepción y Sistemas Inteligentes, Facultad de Ingeniería, Universidad del Valle, Cali-Colombia, jean.diaz@correounivalle.edu.co

4 M.Sc. en Instrumentación Física, Grupo de Investigación Khimera, Facultad de Ingeniería, Fundación Universitaria Católica Lumen Gentium, CaliColombia, pavelez@unicatolica.edu.co 
Evaluación del aporte de la covarianza de las señales electroencefalográficas a las interfaces cerebrocomputador de imaginación motora para pacientes con lesiones de médula espinal

\section{Resumen}

Las interfaces cerebro-computadora no invasivas basadas en EEG de imaginación motora (miBCI) prometen restaurar efectivamente el control motor a pacientes con discapacidades motoras, por ejemplo, aquellos con lesión de la médula espinal (LME). Sin embargo, todavía es necesario investigar las miBCI, con fines de rehabilitación, para este tipo de pacientes que utilizan dispositivos de adquisición de señales EEG de bajo costo, tales como Emotiv EPOC. En este trabajo, se describe en detalle y se comparan diez arquitecturas miBCI basadas en información de covarianza de señales EEG, adquiridas con Emotiv EPOC, para la decodificación de intención de mano abierta y cerrada en tres sujetos control y dos pacientes con LME cervical. Cuatro de estas diez miBCI usan información de covarianza para construir filtros espaciales y el resto usa la información covarianza como una representación directa de las señales EEG, permitiendo la manipulación directa mediante geometría de Riemann. Como resultado, se encontró que, a pesar de que todas las arquitecturas miBCI tienen una precisión general por encima del nivel de azar, las que utilizan la covarianza como representación directa de las señales EEG junto con clasificadores lineales, superan las miBCI que usan la covarianza para el filtrado espacial, tanto en sujetos de control como en pacientes con LME. Estos resultados sugieren un alto potencial de las miBCI basadas en la geometría de Riemann para la rehabilitación de pacientes con LME, utilizando dispositivos de adquisición de EEG de bajo costo.

\section{Palabras clave}

Interfaces cerebro-computador, imaginación motora, geometría de la información, filtrado espacial, lesión de médula espinal.

\section{Abstract}

Non-invasive EEG-based motor imagery brain-computer interfaces (miBCIs) promise to effectively restore the motor control of motor-impaired patients with conditions that include Spinal Cord Injury (SCI). Nonetheless, miBCIs should be further researched for this type of patients using low-cost EEG acquisition devices, such as the Emotiv EPOC, for home rehabilitation purposes. In this work, we describe in detail and compare ten miBCI architectures based on covariance information from EEG epochs. The latter were acquired with Emotiv EPOC from three control subjects and two SCI patients in order to decode the close and open hand intentions. Four out of the ten miBCIs use covariance information to create spatial filters; the rest employ covariance as a direct representation of the EEG signals, thus allowing the direct manipulation by Riemannian geometry. We found that, although all the interfaces present an overall accuracy above chance level, the miBCIs that use covariance as a direct representation of the EEG signals together with linear classifiers outperform miBCIs that use covariance for spatial filtering, both in control subjects and SCI. These results suggest the high potential of Riemannian geometry-based miBCIs for the rehabilitation of SCI patients with low-cost EEG acquisition devices.

\section{Keywords}

Brain-computer interfaces, Motor imagery, Information geometry, Spatial filters, Spinal cord injury. 
Evaluación del aporte de la covarianza de las señales electroencefalográficas a las interfaces cerebrocomputador de imaginación motora para pacientes con lesiones de médula espinal

\section{INTRODUCCIÓN}

Los pacientes con lesiones medulares cervicales presentan grandes retos en la recuperación de las funciones sensitivas y motoras, a nivel de miembros superiores durante la fase aguda y subaguda [1], lo que limita considerablemente su independencia en la realización de tareas diarias [2], [3]. Aun cuando las terapias físicas y ocupacionales son claves en el proceso de recuperación, el paciente, en la mayoría de los casos, se encuentra en una situación de alta dependencia, tanto de los terapeutas como de sus familiares en la ejecución de tareas de motricidad fina $\mathrm{y}$ gruesa. Como consecuencia de esta dependencia, los pacientes dejan de asistir a los encuentros en las clínicas de rehabilitación y, en casos extremos, llegan a perder el interés por la realización de las sesiones terapéuticas, incluso en la comodidad de su hogar. Por esto, en los últimos años, un tipo particular de tecnologías de asistencia basadas en señales del cerebro llamadas [4] interfaces cerebro-computador, BCI, (por sus siglas en inglés: Brain-Computer Interface) [5], [6], permiten comandar dispositivos externos como exomanos, computadores o sillas de ruedas. Esto ha llamado la atención de expertos en rehabilitación de pacientes con limitaciones motrices, ya que su uso por parte del paciente puede complementar las sesiones terapéuticas e, incluso en algunos casos, motivar la realización de las mismas en los que han perdido el interés. Más aun, recientemente, el uso de esta tecnología por pacientes con paraplejia ha demostrado sus beneficios clínicos en procesos de rehabilitación [7], permitiendo pasar de una lesión completa (pérdida total de las funciones sensitivas y motoras por debajo de la lesión) a incompleta (pérdida parcial de las funciones sensitivas y motoras por debajo de la lesión) [4]. Esto ha motivado la investigación y desarrollo en este tipo de tecnologías para rehabilitación de miembros superiores en pacientes con lesiones cervicales.

En la actualidad, existen diversos tipos de BCI, dependiendo de la técnica de adquisición, del tipo modulación cerebral particular que se utilice y del problema de rehabilitación propio en cada paciente. En particular, la interfaz cerebro-computador no invasiva electroencefalográfica, EEG, basada en imaginación motora, miBCI (del inglés: motor imagery Brain-Computer Interface), es considerada una de las más apropiadas para rehabilitación motriz de miembros superiores [8], no solo en pacientes con lesiones medulares, sino también en pacientes con déficit motores debido a otras causas, por ejemplo, en pacientes con accidente cerebro vascular [9]. De acuerdo con [10], esto se debe a que mediante la imaginación motora es posible, no solo incrementar las capacidades de agarre y la fortaleza muscular, sino también disminuir el tiempo de realización del movimiento, mejorar la variabilidad en la ejecución de la trayectoria del mismo y corregir anormalidades en los patrones de actividad cerebral, incrementados en algunos casos después de la LME [11].

Las miBCI generalmente constan de dos fases [12]-[14]. Primero, una fase de calibración o entrenamiento, en la que se recogen segmentos (épocas) de las señales EEG (preferiblemente a nivel superficial) asociadas a dos o más intenciones motoras imaginadas, las cuales permiten entrenar un modelo de clasificación de señales EEG. Después, una fase de retroalimentación, en la que se utiliza el modelo entrenado para clasificar intenciones motoras con base en el protocolo de entrenamiento propuesto en la fase de calibración (ver Fig. 1). Para la construcción de estos modelos de clasificación se hace uso principalmente de técnicas de procesamiento digital de señales [15] y de reconocimiento de patrones [16], [17] que permiten el tratamiento adecuado de la no estacionariedad y la baja relación señal 
Evaluación del aporte de la covarianza de las señales electroencefalográficas a las interfaces cerebrocomputador de imaginación motora para pacientes con lesiones de médula espinal

ruido de las señales EEG superficiales [18], [19] y la efectiva discriminación de diferentes tareas mentales, respectivamente.

En los últimos años, dos técnicas que explotan la información de covarianza de las señales EEG han demostrado una destacable capacidad discriminante en un sinnúmero de retos internacionales sobre bancos de datos disponibles por parte de la comunidad científica para fines de comparación [20]-[24]. Las primeras utilizan la información de covarianza para la construcción de filtros supervisados espaciales, denominadas patrones comunes espaciales [23], [25], junto con técnicas de clasificación clásicas como el análisis discriminante cuadrático/lineal [26] o máquinas de vectores de soporte [17], [27].

En la otra técnica, la información de covarianza es tratada como patrón característico de la señal EEG y su tratamiento se realiza en una variedad de Riemann [14], [21], [28]-[31]. A pesar de los resultados sobresalientes de estos dos tipos de técnicas en los retos internacionales, en su mayoría con sujetos de control sin ningún de tipo de lesión medular o daño cerebral, este tipo de técnicas ha sido poco evaluado, utilizando dispositivos de bajo costo para EEG superficial, como el Emotiv EPOC [32] en pacientes con lesiones medulares a nivel cervical. En [33] se ha mostrado que el Emotiv EPOC es un dispositivo habilitado para el desarrollo, no solo de miBCI, sino también de BCI relacionada a otro tipo de eventos como los auditivos [34], con capacidades similares a las de equipos costosos para la adquisición de EEG superficial para investigación avanzada. Por esto, la contribución de este estudio está dirigido a determinar arquitecturas más simples y robustas, que puedan ser utilizadas en equipos de bajo costo de adquisición de señales EEG como el Emotiv EPOC, para masificar su uso en aplicaciones de rehabilitación orientadas a personas con limitaciones motrices. Por lo tanto, se plantea como hipótesis que el desempeño de las BCI que utilizan la información de covarianza de las señales EEG mejora más cuando dicha información se utiliza para representar las señales EEG junto con modelos lineales de clasificación, que cuando se las utiliza con modelos no lineales o para procesos de filtrado.

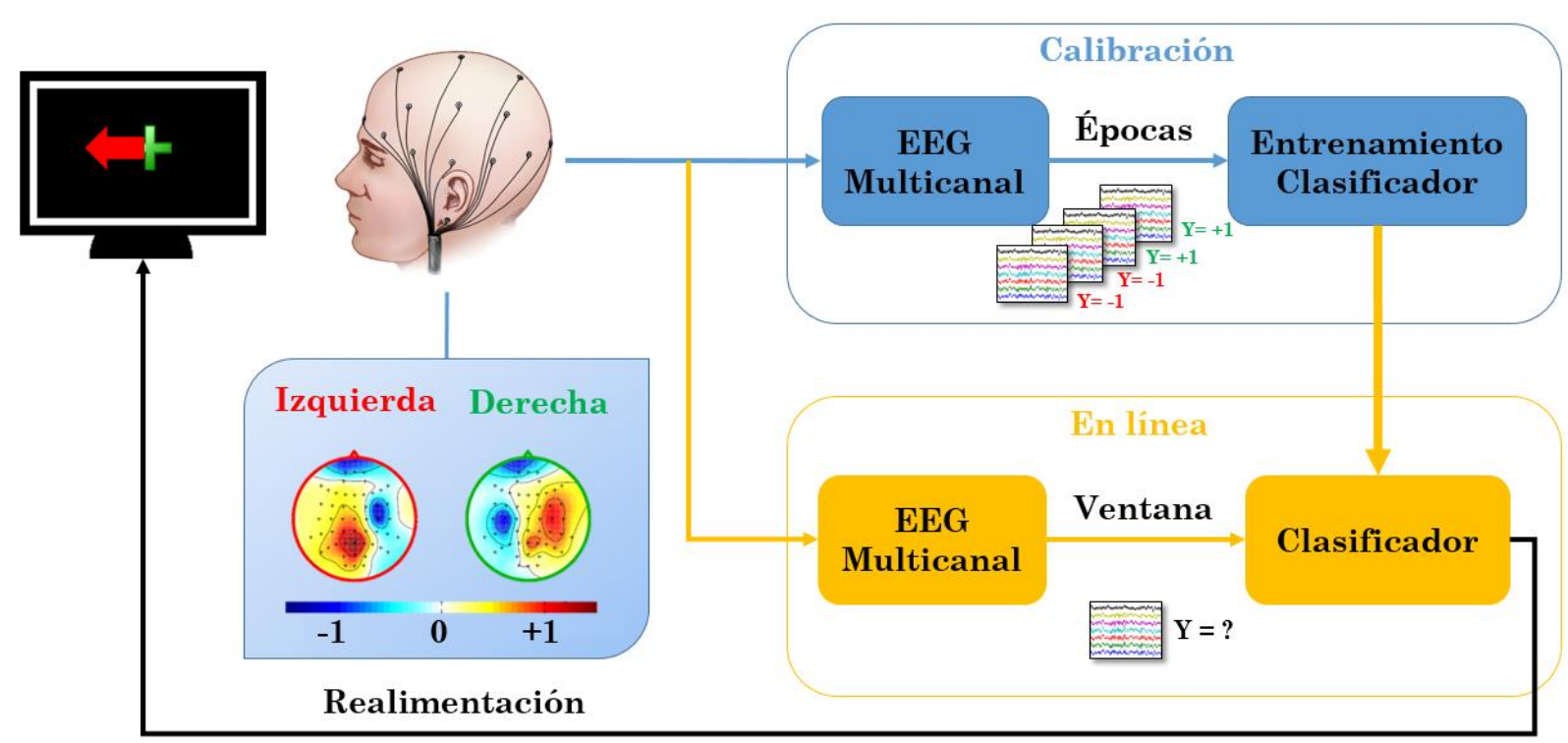

Fig. 1. Visión general de un sistema miBCI utilizando técnicas de procesamiento de señales y reconocimiento de patrones. Fuente: autores. 


\section{Evaluación del aporte de la covarianza de las señales electroencefalográficas a las interfaces cerebro-}

computador de imaginación motora para pacientes con lesiones de médula espinal

Para desarrollar la hipótesis, en este trabajo se diseñaron diez arquitecturas de BCI asociadas a dos estados mentales (imaginación de apertura y cierre de mano), cuatro de ellas utilizaron la información de covarianza de las señales EEG (basados en patrones comunes espaciales) para procesos de filtrado y las otras seis (tres con clasificador lineal y tres con clasificador no lineal) utilizaron la misma información como una nueva representación de dichas señales (basados en geometría de Riemann). Las señales fueron adquiridas con ayuda del Emotiv EPOC [32], [34] en tres sujetos de control y dos pacientes con lesiones cervicales, mediante un protocolo de imaginación motora guiado por marcadores fijos en el tiempo. El desempeño de cada arquitectura se calculó como la precisión de clasificación correcta en un esquema de validación cruzada, teniendo en cuenta todas las muestras, independiente del sujeto. Adicionalmente se mostró el desempeño de cada una de las arquitecturas mediante el área bajo la curva ROC (AUC) por sujeto, con el fin de encontrar variaciones de los resultados con la condición de los sujetos (sano o con LME).

Por lo tanto, en la sección 2 el documento describe el protocolo experimental, los fundamentos matemáticos de los patrones comunes espaciales de la geometría de Riemann y los clasificadores considerados en ambos casos, finalizando con las arquitecturas definitivas para las miBCI. Por último, en la sección 3, se detallan las pruebas llevadas a cabo y se comparan las diferentes arquitecturas para cada sujeto de experimentación.

\section{MATERIALES Y MÉTODOS}

En este estudio se adquirieron segmentos (épocas) de señales EEG asociadas a imaginación de mano cerrada e imaginación de mano abierta de cinco sujetos con edades entre 22 y 56 años, dos de ellos con lesión cervical (S1 y S2) y tres sin ningún tipo de lesión (S3, S4, y S5) (Ver Tabla 1.). De acuerdo con los especialistas médicos, ninguno de los sujetos de prueba presentó limitaciones cognitivas ni daño cerebral que impidiera la participación de los sujetos de experimentación. Asimismo, los sujetos dieron consentimiento informado respecto a las pruebas realizadas y los datos adquiridos.

Cada sujeto se ubicó frente a una pantalla de 55 pulgadas a $1.5 \mathrm{~m}$ de distancia. En la pantalla se desplegaron en forma aleatoria flechas orientadas hacia la derecha (indicando imaginación de mano cerrada) y hacia la izquierda (indicando imaginación de mano abierta). En el caso de los sujetos con lesiones cervicales, se utilizó la silla de ruedas propia de cada sujeto. Para la captura de las señales EEG se usó el Emotiv EPOC el cual consta de 14 canales (AF3, F7, F3, FC5, T7, P7, O1, O2, $\mathrm{P} 8, \mathrm{~T} 8, \mathrm{FC} 6, \mathrm{~F} 4, \mathrm{~F} 8, \mathrm{AF} 4)$ con frecuencia de muestreo de $128 \mathrm{~Hz}$.

Tabla 1. Descripción de sujetos de experimentación. DL: después de la lesión.

\begin{tabular}{cccc}
\hline Tipo & Sujeto & Nivel de la lesión & Edad \\
\hline $\begin{array}{c}\text { Con lesión } \\
\text { medular }\end{array}$ & S1 & C5-C6-C7 (ASIA B) & 33 (1 año DL) \\
& S2 & C3-C4 (ASIA B) & $56(20$ \\
Sin lesión & S3 & N/A & 32 \\
medular & S4 & N/A & 22 \\
& S5 & N/A & 29 \\
\hline
\end{tabular}


Evaluación del aporte de la covarianza de las señales electroencefalográficas a las interfaces cerebrocomputador de imaginación motora para pacientes con lesiones de médula espinal

Por cada sujeto se realizaron 20 sesiones y durante cada sesión se ejecutaron 10 ensayos. Cada ensayo tuvo una duración de 8 segundos y solo se desplegó una de las flechas a la vez. El esquema de eventos durante cada ensayo se puede observar en la Fig. 2. Las épocas de las señales EEG para la imaginación de mano cerrada o abierta se extrajeron desde los $2 \mathrm{~s}$ hasta $\operatorname{los} 6 \mathrm{~s}$. En cada sesión se siguió un protocolo de asepsia que consistió en limpiar el cuero cabelludo de los sujetos de experimentación con agua destilada y se aseguró que los canales del Emotiv EPOC presentaran alta calidad de contacto con ayuda del Software Emotiv Test Bench v.15.0.3. Las épocas de las señales EEG extraídas se procesaron mediante un filtro Butterworth de $5^{\circ}$ orden pasa banda con frecuencias de corte de $8-30 \mathrm{~Hz}$, con el fin de filtrar de forma plana las componentes sensomotoras asociadas a la imaginación de mano cerrada y abierta [35]. Se concibieron dos tipos de interfaces cerebro computador que aprovecharon la información de covarianza entre los canales de las épocas EEG. El primero de ellos utilizó esta información para la construcción de filtros espaciales óptimos que mejoraron la relación señal ruido, y el otro tipo de utilizó esta misma información como una nueva representación de las épocas de señales EEG, la cual pudo ser manipulada sobre una variedad Riemanniana para la construcción de filtros y clasificadores. La descripción matemática de las dos técnicas mencionadas anteriormente se lleva a cabo en las subsecciones siguientes.

\subsection{Patrones comunes espaciales para miBCI}

Anteriormente se mostró que las épocas de las señales EEG fueron filtradas temporalmente. Ahora, dada la naturaleza espacial de esta señal, se pueden concebir filtros espaciales para poder realzar características que permitan discriminar entre los dos tipos de imaginación motora en este tipo de señales. De hecho, los filtros espaciales han sido ampliamente utilizados en investigaciones en neurociencia $\mathrm{y}$ neuroingeniería en todo el mundo [36]. Se utilizan principalmente para aumentar la relación señal ruido de la señal EEG [37].

$\mathrm{El}$ filtrado Laplaciano, el filtrado basado en análisis de componentes principales $(\mathrm{PCA}) \quad \mathrm{o}$ análisis de componentes independientes (ICA) son algunos filtros espaciales comúnmente utilizados. La característica principal de este tipo de filtros es que son no supervisados, es decir, que no tienen en cuenta la categorización (imaginación derecha o izquierda) de la señal EEG.

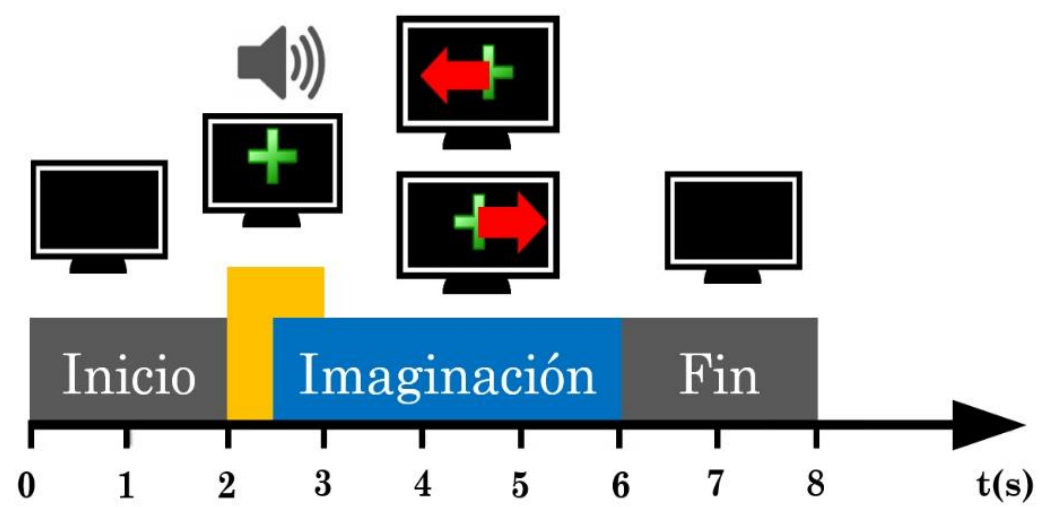

Fig. 2. Esquema de tiempo para imaginación motora. El segmento en azul corresponde al periodo de tiempo en el que se extrae una época de señal EEG asociado con el tipo de movimiento que debe realizarse con base en la dirección de las flechas. Fuente: autores. 
Evaluación del aporte de la covarianza de las señales electroencefalográficas a las interfaces cerebrocomputador de imaginación motora para pacientes con lesiones de médula espinal

Por el contrario, existe un tipo especial de filtros denominados patrones comunes espaciales (CSP) [23], los cuales se construyen de forma supervisada, esto es, teniendo en cuenta la clase a la que pertenece cada segmento de señal EEG. Para la obtención de este filtro se parte del problema de maximización del cociente Rayleigh [38] asociado a las matrices covarianza de los segmentos de señal relacionados con las imaginaciones motoras. Sea E1 y E2 segmentos de señal EEG de $\mathrm{N}$ canales $\mathrm{y} h$ muestras para imaginación de movimiento de mano cerrada (clase 1) y mano abierta (clase 2). Las matrices de covarianza C1 y $\mathrm{C} 2$ asociadas a E1 y E2 respectivamente se obtienen mediante la siguiente expresión (1):

$$
C_{i}=\frac{E_{i} E_{i}^{T}}{\operatorname{tr}\left(E_{i} E_{i}^{T}\right)}, \quad i=1,2
$$

En (1) $\operatorname{tr}($.$) es el operador traza, esto es,$ la suma de los elementos de la diagonal principal de una matriz. Si $\mathrm{Ci}$ se calcula con $\mathrm{h}>\mathrm{N}$ (para este caso $\mathrm{N}=14 \mathrm{y} \mathrm{h}=512$ ), entonces la probabilidad de que $\mathrm{Ci}$ sea invertible aumenta, garantizándose así que la matriz no solo sea simétrica sino también definida positiva. Ahora bien, si $\overline{C_{1}}$ y $\overline{\mathrm{C}_{2}}$ son matrices promedios de covarianza para la clase 1 y 2 respectivamente, en la teoría de filtros espaciales es posible construir de forma supervisada un filtro espacial W con ayuda del cociente de Rayleigh (2):

$$
J(w)=\frac{w^{\mathrm{T}} \overline{C_{1}} w}{w^{\mathrm{T}} \overline{\mathrm{C}_{2}} w}, \quad w \in \mathbb{R}^{\mathrm{N}}
$$

Los $\mathrm{w}^{*}$ que maximizan este cociente se obtienen resolviendo el siguiente problema de optimización sin restricción (3):

$$
\mathrm{w}^{*}=\arg \max _{\mathrm{w}} \mathrm{J}(\mathrm{w})
$$

Nótese que si $\mathrm{w}^{*}$ maximiza el Cociente de Rayleigh entonces $\mathrm{cw}^{*}$ también maximiza este cociente dando el mismo valor. Luego entonces el problema de optimización en (3) puede ser planteado como un problema con restricción (4):

$$
\begin{gathered}
\mathrm{w}^{*}=\underset{\mathrm{w}}{\arg \max _{\mathrm{w}} \mathrm{w}^{\mathrm{T}} \overline{\mathrm{C}_{1}} \mathrm{w}} \\
\text { s.t. } \mathrm{w}^{\mathrm{T}} \mathrm{C}_{2} \mathrm{w}=\mathrm{c}
\end{gathered}
$$

Básicamente (4) es un problema de programación cuadrática con restricción cuadrática, el cual puede ser resuelto de forma iterativa, utilizando programación secuencial cuadrática (SQP) [39] o de forma analítica, utilizando la teoría del multiplicador de Lagrange. Sin embargo, al aplicar SQP de forma directa permite encontrar un solo punto crítico, dando a lo sumo un solo filtro espacial, es decir que la señal se reducirá a un solo canal después de filtrar. Por esta razón, se utiliza la teoría del multiplicador de Lagrange para encontrar $\mathrm{N}$ puntos críticos. Para esto, el problema de maximización (4) se puede formular como un problema de minimización con restricción mediante (5).

$$
\begin{gathered}
\mathrm{w}^{*}=\arg \min _{\mathrm{w}}-\mathrm{w}^{\mathrm{T}} \overline{\mathrm{C}_{1}} \mathrm{w} \\
\text { s.t. } \mathrm{w}^{\mathrm{T}} \mathrm{C}_{2} \mathrm{w}=\mathrm{c}
\end{gathered}
$$

La función de Lagrange asociada al anterior problema es (6):

$$
L(w, \lambda)=-w^{T} \overline{C_{1}} w+\lambda\left(w^{T} \overline{C_{2}} w-c\right)
$$

Aplicando condiciones de primer orden, se deriva (6) respecto a $\mathrm{w}$ y se iguala a cero. Ver (7):

$$
\nabla_{\mathrm{w}} \mathrm{L}\left(\mathrm{w}^{*}, \lambda^{*}\right)=0
$$

Dado que C1 y C2 son simétricas, sustituyendo (6) en (7), se obtiene (8):

$$
-2 \mathrm{w}^{* \mathrm{~T}} \overline{\mathrm{C}_{1}}+\lambda^{*}\left(2 \mathrm{w}^{* \mathrm{~T}} \overline{\mathrm{C}_{2}}\right)=0
$$

Despejando uno de los sumandos de (8) se obtiene (9).

$$
\overline{\mathrm{C}_{1}} \mathrm{w}^{*}=\lambda^{*} \overline{\mathrm{C}_{2}} \mathrm{w}^{*}
$$


Evaluación del aporte de la covarianza de las señales electroencefalográficas a las interfaces cerebrocomputador de imaginación motora para pacientes con lesiones de médula espinal

(9) es evidentemente un problema de autovalores generalizado [40]. Los multiplicadores de Lagrange, $\lambda^{*}$, que satisfacen (9) son en consecuencia los autovalores generalizados. $\mathrm{Si} \quad \overline{C_{2}}$ es invertible el problema de autovalores generalizados puede ser planteado como un problema normal de autovalores (10):

$$
\left(\left(\overline{\mathrm{C}_{2}}\right)^{-1} \overline{\mathrm{C}_{1}}\right) \mathrm{w}^{*}=\lambda^{*} \mathrm{w}^{*}
$$

Cuando $\overline{C_{2}}$ es invertible los filtros espaciales pueden ser obtenidos mediante (10). Sin embargo, si $\overline{C_{2}}$ no es invertible es posible que el costo máximo obtenido de (2) sea ilimitado, por lo anterior, se impone otra restricción sobre la norma de $\mathrm{w}$ al problema de optimización inicial, ver (11):

$$
\begin{gathered}
\mathrm{w}^{*}=\underset{\mathrm{w}}{\arg \min _{\mathrm{w}}-\mathrm{w}^{\mathrm{T}} \overline{\mathrm{C}_{1}} \mathrm{w}} \\
\text { s.t. } \mathrm{w}^{\mathrm{T}} \mathrm{C}_{2} \mathrm{w}=\mathrm{c} \\
\mathrm{w}^{\mathrm{T}} \mathrm{w}=\mathrm{d}
\end{gathered}
$$

Para este caso la Lagrangiana viene dado por (12):

$$
\begin{gathered}
\mathrm{L}\left(\mathrm{w}, \lambda_{1}, \lambda_{2}\right)=-\mathrm{w}^{\mathrm{T}} \overline{\mathrm{C}_{1}} \mathrm{w}+\lambda_{1}\left(\mathrm{w}^{\mathrm{T}} \overline{\mathrm{C}_{2}} \mathrm{w}-\mathrm{c}\right) \\
+\lambda_{2}\left(\mathrm{w}^{\mathrm{T}} \mathrm{w}-\mathrm{d}\right)
\end{gathered}
$$

Las soluciones deben satisfacer $\nabla_{\mathrm{w}} \mathrm{L}\left(\mathrm{w}^{*}, \lambda_{1}^{*}, \lambda_{2}^{*}\right)=0$, esto es (13):

$$
-\mathrm{w}^{* \mathrm{~T}} \overline{\mathrm{C}_{1}}+2 \lambda_{1}^{*}\left(\mathrm{w}^{* \mathrm{~T}} \overline{\mathrm{C}_{2}}\right)+\lambda_{2}^{*}\left(\mathrm{w}^{* \mathrm{~T}} \mathrm{I}\right)=0
$$

Reorganizando (13) se obtiene (14):

$$
\begin{gathered}
\mathrm{w}^{* \mathrm{~T}} \overline{\mathrm{C}_{1}}+2 \lambda_{1}^{*} \mathrm{w}^{*}\left(\overline{\mathrm{C}_{2}}+\gamma \mathrm{I}\right)=0 ; \\
\text { Tal que: } \gamma=\frac{\lambda_{2}^{*}}{\lambda_{1}^{*}}
\end{gathered}
$$

Nótese que (14) parece el problema de optimización inicial (5) pero ahora $\overline{C_{2}}$ está acompañado de un término de regularización que permite que sea invertible. Esto permite introducir el problema de optimización de patrones Comunes Espaciales Regularizados (RCSP), el cual en forma general se plantea de la siguiente forma (15):

$$
\begin{gathered}
\mathrm{w}^{*}=\arg \min _{\mathrm{w}}-\mathrm{w}^{\mathrm{T}} \overline{\mathrm{C}_{1}} \mathrm{w} \\
\text { s.t. } \mathrm{w}^{\mathrm{T}}\left(\overline{\mathrm{C}_{2}}\right) \mathrm{w}+\gamma \mathrm{P}(\mathrm{w})=\mathrm{c} ; \\
\mathrm{P}(\mathrm{w})=\mathrm{w}^{\mathrm{T}} \mathrm{Iw}
\end{gathered}
$$

En síntesis, la obtención de un filtro $W \in \mathbb{R}^{N \times N}$ tipo CSP junto con sus versiones regularizadas y no regularizadas se compilan en Tabla 2.

El efecto final del filtro $W \in \mathbb{R}^{\mathrm{N} \times \mathrm{N}}$ aplicado a la señal Ei es la de aumentar la varianza para la mitad de los canales de $\mathrm{Ei}$, mientras que disminuye la misma para el resto de canales para una clase en particular, y viceversa en el caso de otra clase. Por esto suele utilizarse como patrón característico, asociado a Ei (después de ser filtrado), la varianza logarítmica, obtenida mediante la función $<\ln (\circ)>(16)$.

$$
\mathrm{e}_{\mathrm{i}}=<\ln \left(W \mathrm{E}_{\mathrm{i}}\right)>
$$

Nótese que dado que $\mathrm{WE}_{\mathrm{i}} \in \mathbb{R}^{\mathrm{N} \times \mathrm{h}}$, cada componente de ei se obtiene aplicando el logaritmo natural a la varianza de cada canal de WEi, lo que hace que finalmente $\mathrm{e}_{\mathrm{i}} \in \mathbb{R}^{\mathrm{N}}$. El Algoritmo 1 (ver Tabla 3.) describe el procedimiento para la obtención de un vector de varianza logarítmica para una señal EEG, Ex, filtrada espacialmente.

\subsection{Geometría de Riemann para miBCI}

Las matrices de covarianza simétricas y definidas positivas, $\mathrm{Ci}$, pueden ser utilizadas como representación de las señales EEG, Ei. Este tipo de matrices simétricas y definidas positivas pertenecen a una forma particular de variedad matemática conocida como variedad de Riemann, $\mathrm{C}(\mathrm{N})$. En esta variedad matemática, la distancia Riemanniana de dos matrices, $\mathrm{C} 1$ y C2, viene dada por (17):

$$
\delta_{\mathrm{R}}\left(\mathrm{C}_{1}, \mathrm{C}_{2}\right)=\left\|\log \left(\mathrm{C}_{1}^{-1} \mathrm{C}_{2}\right)\right\|_{\mathrm{F}}=\left[\sum_{\mathrm{n}=1}^{\mathrm{N}} \log ^{2} \beta_{\mathrm{n}}\right]^{1 / 2}
$$


Evaluación del aporte de la covarianza de las señales electroencefalográficas a las interfaces cerebrocomputador de imaginación motora para pacientes con lesiones de médula espinal

Tabla 2. Programas no lineales para CSP regularizados y no regularizados. Fuente: autores

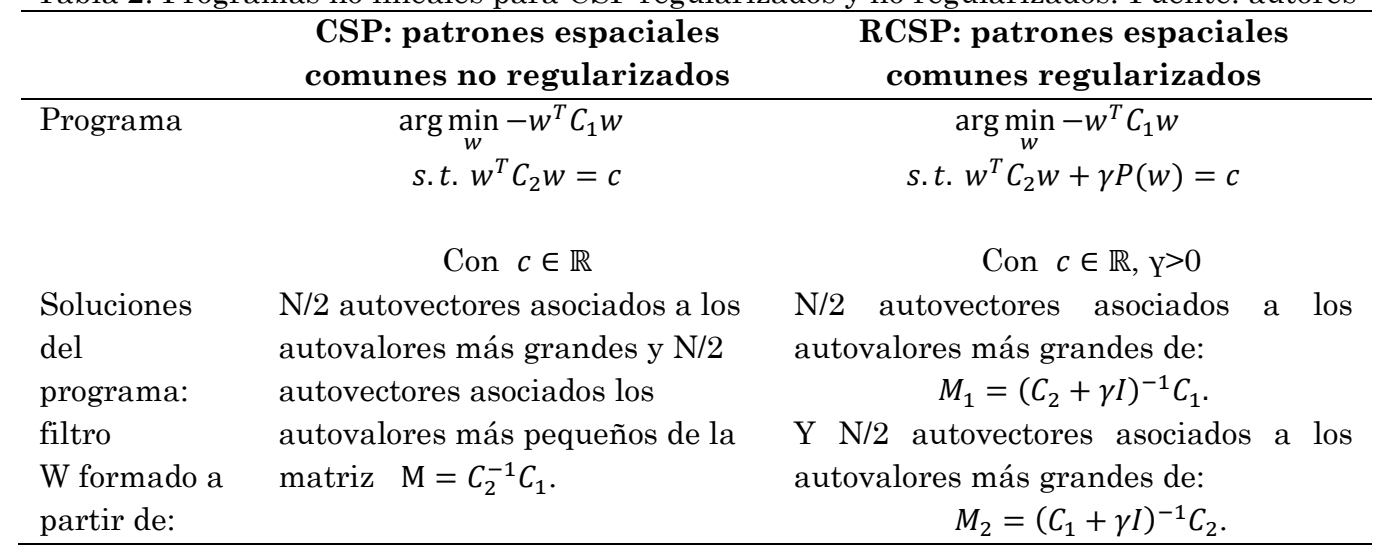

Tabla 3. Descripción de algoritmo para obtención de vectores de varianza logarítmica. Fuente: autores.

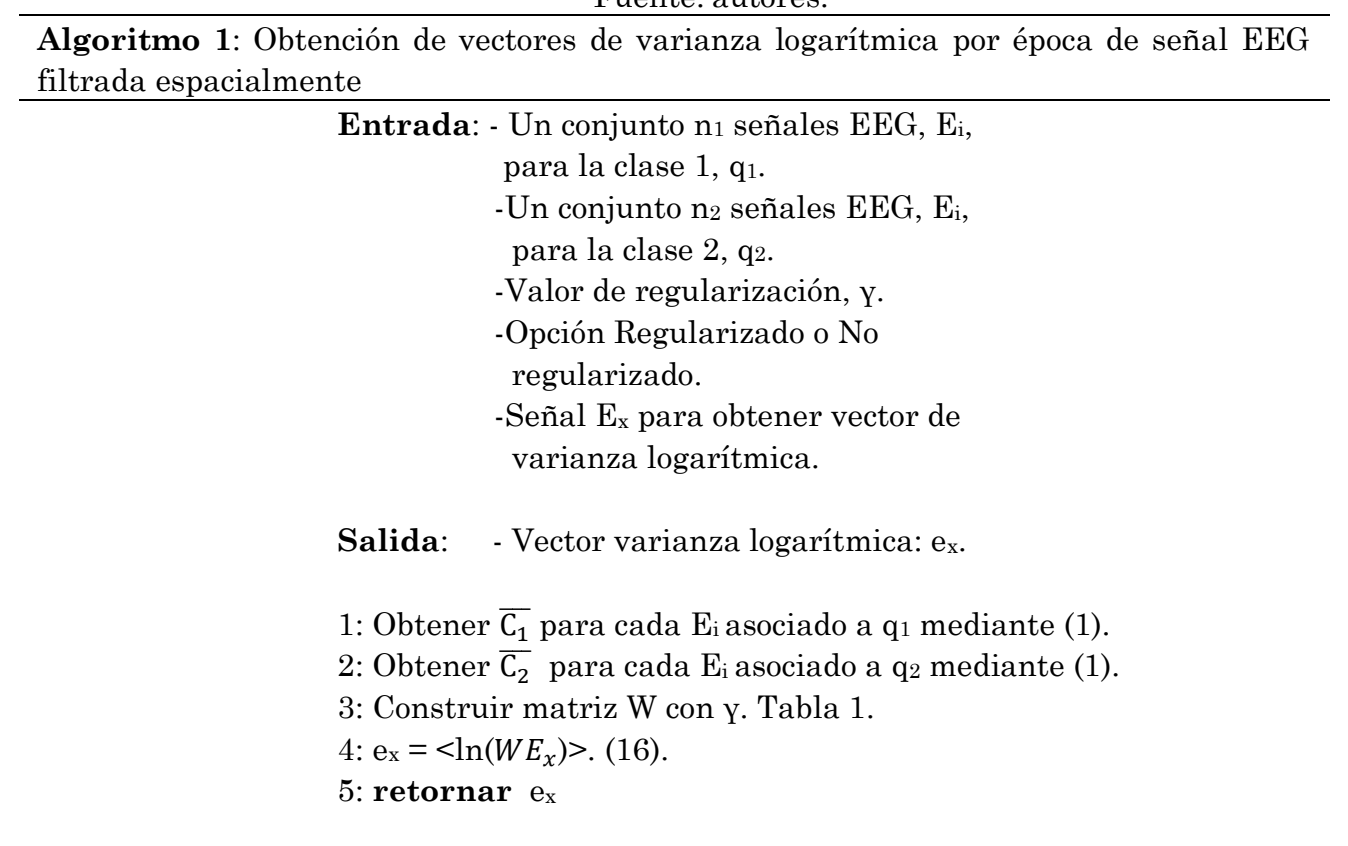

Donde $\beta_{n}$ son los autovalores de $C_{1}^{-1} C_{2}$. $\log \left(\mathrm{C}=C_{1}^{-1} C_{2}\right)$ es el logaritmo de una matriz, el cual se calcula, con ayuda de la descomposición en valores singulares de C mediante el siguiente producto de matrices: $U(\operatorname{diag}[\ln (\beta 1), \ldots, \ln (\beta \mathrm{n})]) \mathrm{U}^{T}$, donde $\mathrm{U}$ es la matriz de autovectores de $\mathrm{C}$. $\|\cdot\|_{F}$ es la norma de frobenious de una matriz, la cual se obtiene como la raíz cuadrada de la traza del producto entre la matriz y su transpuesta.

Para utilizar esta distancia en un problema de clasificación de señales Ei, se obtienen matrices promedio, una para la clase $1, \overline{\overline{C_{1}}}, y$ otra para la clase $2, \overline{\overline{C_{2}}}$. La discriminación de una matriz $\mathrm{C}$ se hace por menor distancia a las matrices promedio anteriores. Para encontrar estas matrices promedio, no es posible utilizar la fórmula de media de aritmética de matrices convencional, utilizada en la sección anterior, ya que las matrices de covarianza no residen en el espacio euclídeo sino en una variedad Riemanniana. En este espacio la matriz promedio asociado a M1, matrices de covarianza para la clase 1 y M2 matrices de covarianza para la clase 2, se obtiene resolviendo los siguientes 
Evaluación del aporte de la covarianza de las señales electroencefalográficas a las interfaces cerebrocomputador de imaginación motora para pacientes con lesiones de médula espinal

problemas de optimización respectivamente (18) y (19):

$$
\begin{aligned}
& \overline{\overline{\mathrm{C}_{1}}}=\arg \min _{C \in C(\mathrm{n})} \sum_{\mathrm{i}=1}^{\mathrm{M}_{1}} \delta_{\mathrm{R}}\left(\mathrm{C}, \mathrm{C}_{\mathrm{i} 1}\right) \\
& \overline{\overline{\mathrm{C}_{2}}}=\arg \min _{\mathrm{C} \in \mathrm{C}(\mathrm{n})} \sum_{\mathrm{i}=1}^{\mathrm{M}_{2}} \delta_{\mathrm{R}}\left(\mathrm{C}, \mathrm{C}_{\mathrm{i} 2}\right)
\end{aligned}
$$

No hay una forma explícita para resolver el problema anterior, pero existen formas iterativas para estimarlo, utilizando el concepto de espacio tangente. Con ayuda del logaritmo Riemanniano entre dos matrices, $\operatorname{LogR}(\mathrm{C}, \mathrm{Ci})$, se proyecta el conjunto de datos completo, $\mathrm{Ci}$ $(\mathrm{i}=1 . . \mathrm{M})$, matrices de covarianza, en el espacio tangente de C. En este espacio, por cierto, de propiedades euclidianas, el promedio puede obtenerse mediante media aritmética. Finalmente, se reproyecta este promedio obtenido al espacio SPD mediante la exponencial Riemanniana entre dos matrices, ExpR (C, Ci). Después de algunas iteraciones, se obtiene la media geométrica de las matrices SPD. Las iteraciones se detienen cuando se ha realizado un número determinado de iteraciones o cuando la distancia de Frobenius de la proyección tangente promedio se hace estacionaria. Ver Algoritmo 2 (ver Tabla 4.)

Las expresiones matemáticas para el logaritmo y exponencial Riemanniano para proyección y reproyección tangente, respectivamente, se muestran en (20) y
(21). Igualmente, estas operaciones se ilustran en la Fig. 3.

$$
\begin{aligned}
& \mathrm{S}_{\mathrm{i}}=\mathrm{C}^{1 / 2} \log \left(\mathrm{C}^{-1 / 2} \mathrm{C}_{\mathrm{i}} \mathrm{C}^{-1 / 2}\right) \mathrm{C}^{1 / 2} \\
& \mathrm{C}_{\mathrm{i}}=\mathrm{C}^{1 / 2} \operatorname{Exp}\left(\mathrm{C}^{-1 / 2} \mathrm{~S}_{\mathrm{i}} \mathrm{C}^{-1 / 2}\right) \mathrm{C}^{1 / 2}
\end{aligned}
$$

Para la construcción de filtros y de clasificadores en el espacio tangente, la matrices de covarianza proyectadas, las cuales son simétricas, $\mathrm{Si}, \mathrm{j}=\mathrm{Sj}, \mathrm{i}$, pueden vectorizarse utilizando únicamente su parte triangular superior (22), como en [14].

La $\sqrt{2}$ se multiplica a todos los elementos que están por fuera de la diagonal principal de $\mathrm{S}$ para que $\|S\|_{\mathrm{F}}=$ $\|$ vect (S) $\|_{2}$. La desvectorización de vect (S) se realiza de forma contraria. Nótese además vect $(S)$ que tiene $\mathrm{N}(\mathrm{N}+1) / 2$ elementos. Esta forma de vectorización permite la construcción de técnicas de filtrado geodésico (GF) como el propuesto en [29] y descrito en el Algoritmo 3 (ver Tabla 5.). La idea básica de este algoritmo es utilizar el análisis discriminante lineal de fisher para la construcción de un filtro a partir de los $\mathrm{K}$ vectores de fisher de las proyecciones de las matrices de covarianza en el espacio tangente de la media riemanniana de un conjunto de $\mathrm{M}$ matrices de covarianza. Para el caso de señales EEG, este filtro debe obtenerse a partir de todas las épocas tanto de imaginación izquierda como derecha.

$$
\operatorname{vect}(\mathrm{S})=\left[\begin{array}{c}
\mathrm{S}_{(1,1)} \\
\sqrt{2} \mathrm{~S}_{(1,2)} \\
\vdots \\
\sqrt{2} \mathrm{~S}_{(1, \mathrm{~N})} \\
\mathrm{S}_{(2,2)} \\
\sqrt{2} \mathrm{~S}_{(2,3)} \\
\vdots \\
\sqrt{2} \mathrm{~S}_{(2, \mathrm{~N})} \\
\vdots \\
\mathrm{S}_{(\mathrm{N}, \mathrm{N})}
\end{array}\right]
$$


Evaluación del aporte de la covarianza de las señales electroencefalográficas a las interfaces cerebrocomputador de imaginación motora para pacientes con lesiones de médula espinal

Tabla 4. Descripción de algoritmo para obtención de matriz promedio Riemanniana. Fuente: autores.

Algoritmo 2: Promedio de matrices de covarianza

Entrada: - Un conjunto de $M$ matrices de covarianza, $\mathrm{C}_{\mathrm{i}} \in \mathrm{C}(\mathrm{N})$.

Valor de tolerancia, $\theta$.

Valor máximo de iteraciones, maxIter.

Salida: - Matriz promedio estimada $\overline{\bar{C}}$.

1: Inicializar $\overline{\overline{\mathrm{C}}}^{(0)}=\frac{1}{\mathrm{M}} \sum_{\mathrm{i}=1}^{\mathrm{M}} \mathrm{C}_{\mathrm{i}}$

2: Calcular $\mathrm{S}=\frac{1}{\mathrm{M}} \sum_{\mathrm{i}=1}^{\mathrm{M}} \log _{\mathrm{R}}\left(\overline{\overline{\mathrm{C}}}^{(0)}, \mathrm{C}_{\mathrm{i}}\right)$

$3: \mathrm{k}=0$

4: mientras $\mathrm{k}<\operatorname{maxIter}$ y $\theta>\|\mathrm{S}\|_{F}$

5: $\quad \mathrm{S}=\frac{1}{\mathrm{M}} \sum_{\mathrm{i}=1}^{\mathrm{M}} \log _{\mathrm{R}}\left(\overline{\overline{\mathrm{C}}}^{(\mathrm{k})}, \mathrm{C}_{\mathrm{i}}\right)$

6: $\quad \overline{\overline{\mathrm{C}}}^{(\mathrm{k}+1)}=\operatorname{Exp}_{\mathrm{R}}\left(\overline{\overline{\mathrm{C}}}^{(\mathrm{k})}, \mathrm{S}\right)$

7: $\quad \mathrm{k}=\mathrm{k}+1$.

8: fin

9: $\overline{\overline{\mathrm{C}}}=\overline{\overline{\mathrm{C}}}^{(\mathrm{k})}$

10: retornar $\overline{\bar{C}}$

Tabla 5. Descripción de algoritmo para filtrado geodésico. Fuente: autores.

Algoritmo 3: Filtrado Geodésico (GF)

Entrada: M matrices de covarianza,

$\mathrm{C}_{\mathrm{i}} \in \mathrm{C}(\mathrm{N})$.

- Matriz $C_{x} \in C(N)$ para filtrar.

- Número K de filtros fisher a utilizar.

Salida: Matriz filtrada geodésicamente

$\widetilde{\mathrm{C}_{\mathrm{X}}}$.

1: $\overline{\mathrm{C}}=$ Algoritmo 2( $\left.\mathrm{C}_{\mathrm{i}} \in \mathrm{C}(\mathrm{N})\right)$.

2: repetir $i=1$ hasta $M$

3: $\mathrm{S}_{\mathrm{i}}=\log _{\mathrm{R}}\left(\overline{\mathrm{C}}, \mathrm{C}_{\mathrm{i}}\right)$.

Proyección espacio tangente.

4: $\widetilde{\mathrm{S}_{1}}=$ concatenar $\left[\operatorname{vect}\left(\mathrm{S}_{\mathrm{i}}\right)\right]$.

Vectorización mediante (22).

5: fin

$6: \widetilde{W}=\operatorname{LDA}\left(\widetilde{S_{1}}\right)$.

Obtención de los vectores fisher.

$7: \widetilde{\mathrm{W}_{\mathrm{R}}}=\left[\widetilde{\mathrm{W}_{1}}, \widetilde{\mathrm{W}_{2}}, \ldots, \widetilde{\mathrm{W}_{\mathrm{K}}}\right] \in \mathbb{R}^{\frac{\mathrm{N}}{2}(\mathrm{~N}+1) \times \mathrm{K}}$.

Selección de $\mathrm{K}$ vectores.

8: $\mathrm{S}_{\mathrm{X}}=\log _{\mathrm{R}}\left(\overline{\mathrm{C}}, \mathrm{C}_{\mathrm{x}}\right)$

9: $\widetilde{S_{X}}=\widetilde{W_{R}}\left({\widetilde{W_{R}}}^{T}{\widetilde{W_{R}}}^{-1}{\widetilde{W_{R}}}^{T} \operatorname{vect}\left(S_{x}\right)\right.$.

Filtrado.

10: $\widetilde{\mathrm{C}_{\mathrm{x}}}=\operatorname{Exp}_{\mathrm{R}}\left(\overline{\mathrm{C}}\right.$, unvect $\left.\left(\widetilde{\mathrm{S}_{\mathrm{x}}}\right)\right)$

11: retornar $\widetilde{\mathrm{C}_{\mathrm{x}}}$ 
Evaluación del aporte de la covarianza de las señales electroencefalográficas a las interfaces cerebrocomputador de imaginación motora para pacientes con lesiones de médula espinal

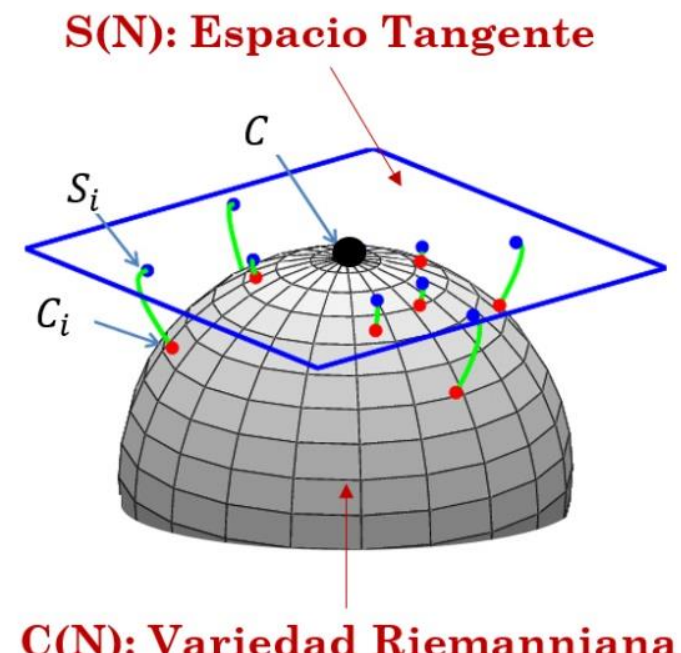

Fig. 3. Espacio tangente de una matriz de covarianza C, la cual pertenece a la variedad Riemanniana $\mathrm{C}(\mathrm{N})$. Si es la proyección de $\mathrm{Ci}$ en el espacio tangente de C. Fuente: autores.

\subsection{Clasificadores}

Los vectores de varianza logarítmica de la sección 2.1 y las vectorizaciones de las proyecciones en el espacio tangente de un punto sobre la variedad de Riemann de la sección 2.2 se asocian a cada una de las señales EEG con su respectiva clase. Para estos patrones es posible desarrollar técnicas de clasificación basadas en análisis discriminante cuadrático (QDA) o lineal (LDA) [26]. Primero, asumiendo que estos patrones poseen una distribución normal, que las probabilidades a priori de ambas clases son iguales y además que la media y covarianza para la clase 1 y 2 viene dado por $\left(\mu_{1}, \Sigma_{1}\right)$ y $\left(\mu_{2}, \sum_{2}\right)$ respectivamente, entonces mediante (23) es posible discriminar de forma cuadrática (QDA) la pertenencia de un patrón ex a una de las clases.

Finalmente, si se asume $\sum_{1}=\sum_{2}=\Sigma$ entonces la discriminación se puede obtener de forma lineal (LDA) mediante (24). Ambas expresiones (23) y (24) permiten clasificar a $e_{x}$ perteneciente a la clase 1 si $y \geq 0$ y a la clase en 2 en caso contrario.

$$
y\left(e_{x}\right)=\left(\mu_{1}-\mu_{2}\right)^{T} \Sigma^{-1}\left[e_{x}-\frac{1}{2}\left(\mu_{1}+\mu_{2}\right)\right]
$$

Las máquinas de vectores de soporte son otra opción robusta para clasificar $\mathrm{e}_{\mathrm{x}}$ mediante (25). $\mathrm{N}_{\mathrm{s}}$ es el número de vectores de soporte obtenidos después de optimizar el problema dual asociado a la maximización del margen de separación entre las clases, $\alpha_{i}$ son los multiplicadores de Lagrange no nulos obtenidos de este mismo problema, di son las etiquetas $(+1$ para la clase 1 y -1 para la clase 2) de los vectores de soporte y $e_{x i}$ son los vectores de soporte los cuales son un subconjunto de los datos de entrenamiento [27]. En este caso $\mathrm{y} \geq+1$ clasifica para la clase 1 e $\mathrm{y} \leq-1$ clasifica para la clase 2 .

$$
y\left(e_{x}\right)=\sum_{i=1}^{N_{s}} \alpha_{i} d_{i} k\left(e_{x}, e_{x i}\right)
$$

$$
\mathrm{y}\left(\mathrm{e}_{\mathrm{x}}\right)=\frac{1}{2} \ln \left(\frac{\left|\sum_{2}\right|}{\left|\sum_{1}\right|}\right)-\frac{1}{2}\left[\left\{\left(\mathrm{e}_{\mathrm{x}}-\quad \mu_{2}\right)^{\mathrm{T}} \sum_{2}^{-1}\left(\mathrm{e}_{\mathrm{x}}-\mu_{2}\right)\right\}-\quad\left\{\left(\mathrm{e}_{\mathrm{x}}-\mu_{1}\right)^{\mathrm{T}} \sum_{1}^{-1}\left(\mathrm{e}_{\mathrm{x}}-\mu_{1}\right)\right\}\right]
$$


Evaluación del aporte de la covarianza de las señales electroencefalográficas a las interfaces cerebrocomputador de imaginación motora para pacientes con lesiones de médula espinal

Por otra parte, si se conserva la representación de las señales EEG en sus matrices de covarianza, con ayuda de (17), (18) y (19) se puede implementar un clasificador riemanniano denominado de mínima distancia a la media (MDM) el cual se expresa en (26). En este caso no se utiliza e $e_{\mathrm{x}}$ sino $\mathrm{C}_{\mathrm{x}}$ asociado a $\mathrm{E}_{\mathrm{x}}$ :

$$
\mathrm{q}=\arg \min _{\mathrm{i} \in\{1,2\}}\left\{\delta_{\mathrm{R}}\left(\overline{\overline{\mathrm{C}}}_{\mathrm{i}}, \mathrm{C}_{\mathrm{x}}\right)\right\}
$$

\subsection{Arquitecturas miBCI basadas en CSP y geometría de Riemann}

Con ayuda de las funciones discriminantes descritas en la sección $2.3 \mathrm{y}$ las técnicas desarrolladas en las secciones 2.1 y 2.2 es posible concebir diez arquitecturas de interfaces cerebrocomputador: cuatro basadas en CSP (Fig. 4) y seis basadas en geometría de Riemann (Fig. 5).

Para la determinación de los parámetros involucrados en los diferentes algoritmos se tomaron valores de referencia encontrados en otros estudios. El parámetro de regularización del Algoritmo 1 (ver Tabla 3) Y se fijó igual 0.0001 (con base en [24]) para garantizar la invertibilidad de las matrices de covarianza en la obtención de los filtros espaciales. El valor de tolerancia $\theta$ para el
Algoritmo 2 (ver Tabla 4) se fijó en 0.001 y el número máximo de iteraciones en 200, tomando como referencia [29], [31]. En el filtrado geodésico, Algoritmo 3 (ver Tabla 5 ), el número $\mathrm{K}$ de vectores de filtros Fisher se fijó en N-1 siguiendo la sugerencia del trabajo reportado en [28]. Finalmente, con base en [27], las máquinas de vectores de soporte fueron entrenadas con un parámetro de regularización igual 100 para penalizar fuertemente los errores de clasificación; y para el caso de la función gaussiana en la máquina de soporte de base radial (Rbf) se tomó una desviación estándar igual nc-1, donde nc es el número de características de los patrones de entrenamiento.

Aun cuando en las miBCI basadas en geometría de Riemann, Fig. 5, pudo considerarse también las técnicas de clasificación mediante los métodos discriminantes planteados en [23] y [24], en este estudio solo se consideró la MDM y las máquinas de vectores de soporte de kernel lineal y gaussiano (Rbf). En trabajos futuros se considerará estos y otro tipo de clasificadores, incluyendo la optimización de hiperparámetros para la obtención de los mejores valores de regularización que maximicen el desempeño de las arquitecturas propuestas.

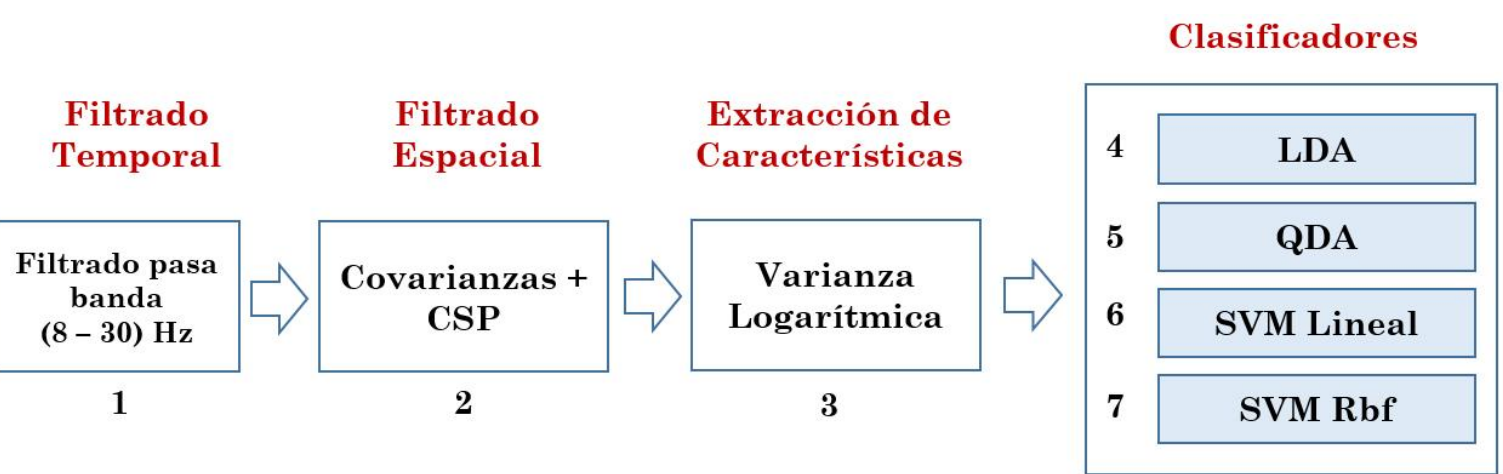

Fig. 4. Cuatro arquitecturas de BCI basadas en CSP: CSP+LDA (1-2-3-4), CSP+QDA (1-2-3-5), CSP+SVM Lineal (1-2-3-6) y CSP+SVM Rbf (1-2-3-7). Fuente: autores. 
Evaluación del aporte de la covarianza de las señales electroencefalográficas a las interfaces cerebrocomputador de imaginación motora para pacientes con lesiones de médula espinal

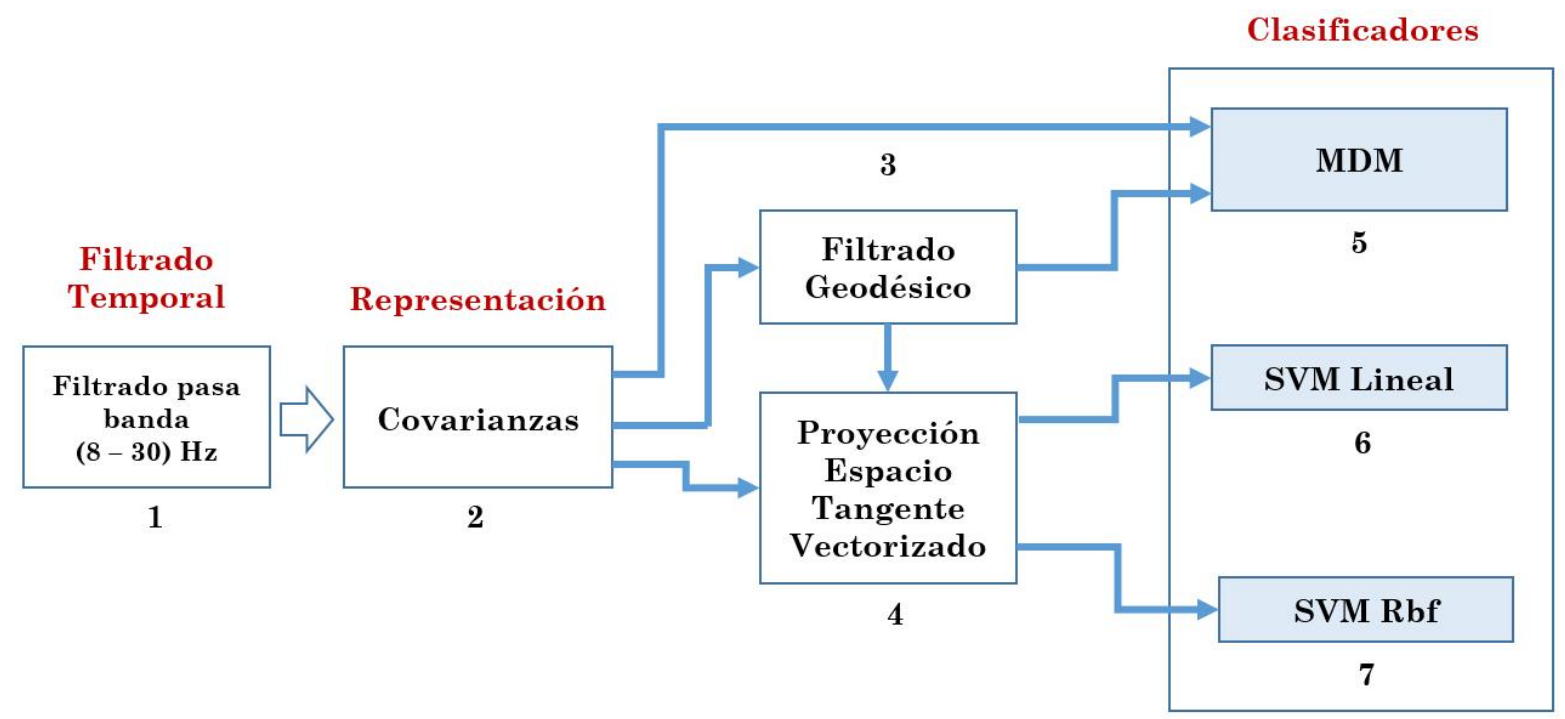

Fig. 5. Seis arquitecturas de miBCI basadas en Geometría de Riemann: RM+MDM (1-2-5), +GF+MDM (1-2-3-5), RM+TS+SVM Lineal (1-2-4-6), RM+TS+SVM Rbf (1-2-4-7), RM+GF+TS+SVM Lineal (1-2-3-4-6), RM+GF+TS+SVM Rbf(1-2-3-4-7). Fuentes: autores.

Es importante mencionar que las arquitecturas miBCI basadas en geometría de Riemann, que hacen uso específico del filtrado geodésico y el clasificador MDM, solo requieren el cómputo iterativo durante el entrenamiento. Durante la ejecución en línea este cómputo no se requiere. En el caso del filtrado geodésico para el cómputo en línea, solo es suficiente con conservar la matriz promedio $\overline{\mathrm{C}}$ y el filtro WR los cuales se obtienen en las líneas 1 y 7 del Algoritmo 3 (ver Tabla 5), respectivamente. En el caso del clasificador MDM, solo habría que almacenar las matrices de covarianza promedio para las clases 1 y 2 obtenidas con ayuda del Algoritmo 2 (ver Tabla 4).

\section{PRUEBAS Y RESULTADOS}

Para evaluar las miBCI basadas en CSP y las basadas en geometría de Riemann descritas en la sección 2, se validó que los promedios de precisión (clasificación correcta) general (incluyendo todos los sujetos) estuvieran por encima del nivel de azar. Estos promedios de precisión fueron obtenidos en un esquema de validación cruzada estratificada con 10 divisiones [41]. Después, con el fin de comparar el desempeño individual de cada una de las arquitecturas para cada uno de los sujetos, se evaluó el área bajo la curva ROC. El banco de datos constó de 200 épocas de EEG de las cuales 100 estuvieron asociadas a imaginación de mano cerrada (flecha derecha) y las otras 100 a mano abierta (flecha izquierda), lo que significa que para los 5 sujetos el total de ensayos fue de 1000 .

Las precisiones de las diferentes arquitecturas sobre todos los ensayos se promediaron a lo largo de las diferentes divisiones del dataset (10 divisiones). Para probar si este desempeño estuvo significativamente por encima del desempeño de un clasificador que asociara cualquier clase a las épocas [42], se estimó el nivel de azar (chance-level, en inglés) como el intervalo de confianza del 0.95 de la inversa de la distribución binomial, en función del número de muestras disponibles (1000) y la probabilidad de las clases (0.5), lo que llevó a un valor de 0.53.

En la Fig. 6 se observa que todos estos valores están por encima del nivel de azar estimado para este caso. Nótese que la arquitectura RM+TS+SVM lineal presenta 
Evaluación del aporte de la covarianza de las señales electroencefalográficas a las interfaces cerebrocomputador de imaginación motora para pacientes con lesiones de médula espinal

la mejor precisión promedio entre todas las arquitecturas. Para determinar cuál o cuáles fueron las mejores miBCI por sujeto, se comparó el desempeño de cada una de estas utilizando el área bajo la curva ROC, AUC [43]. En la Tabla 6 se muestran estos valores obtenidos. Estos mismos datos son graficados en la Fig. 7 para propósitos de comparación visual.

En primer lugar, se observa que independiente del sujeto, los resultados de las miBCI basadas en geometría de Riemann presentaron en promedio un $31 \%$ de desempeño mayor que las basadas en CSP. Sobresalen por su desempeño las siguientes miBCI: $\mathrm{RM}+\mathrm{GF}+\mathrm{MDM}$, $\mathrm{RM}+\mathrm{TS}+\mathrm{SVM} \quad$ lineal $\quad \mathrm{y} \quad$ la $\mathrm{RM}+\mathrm{GF}+\mathrm{TS}+\mathrm{SVM}$ lineal. La superioridad en desempeño de las arquitecturas basadas en Riemann respecto a las basadas en CSP se debe a: i) la estimación de las matrices de covarianza promedio por clase en el caso de CSP no es óptima [44], desde el punto de vista topológico en el espacio de matrices positivas definidas; ii) la obtención de los autovalores y autovectores no tiene en cuenta la dispersión entre clases; iii) la extracción de características mediante el operador de varianza logarítmica se calcula mediante una aproximación de la distancia de Riemann entre matrices de covarianza [44]. Nótese además que las únicas arquitecturas basadas en geometría de Riemann que presentan desempeños inferiores, incluso por debajo que las basadas en CSP, son las que utilizan SVM de kernel gaussiano como clasificador.

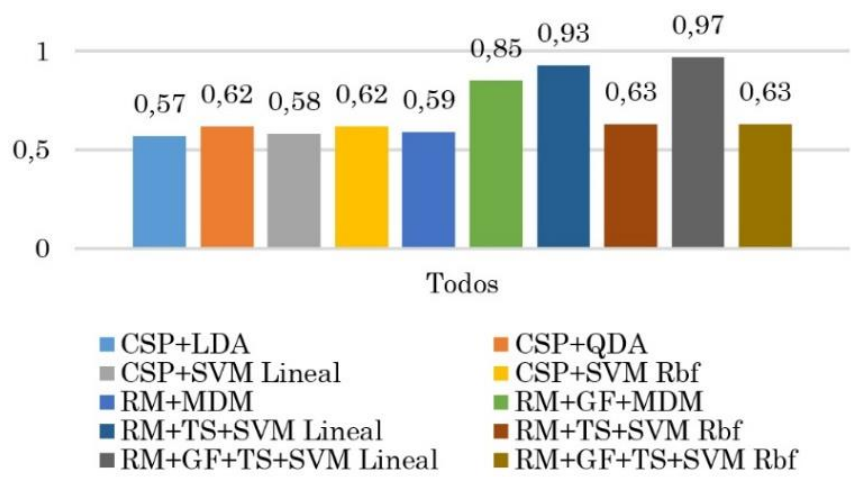

Fig. 6. Precisión general de las arquitecturas miBCI sobre todas las épocas de los sujetos.

Fuente: autores.

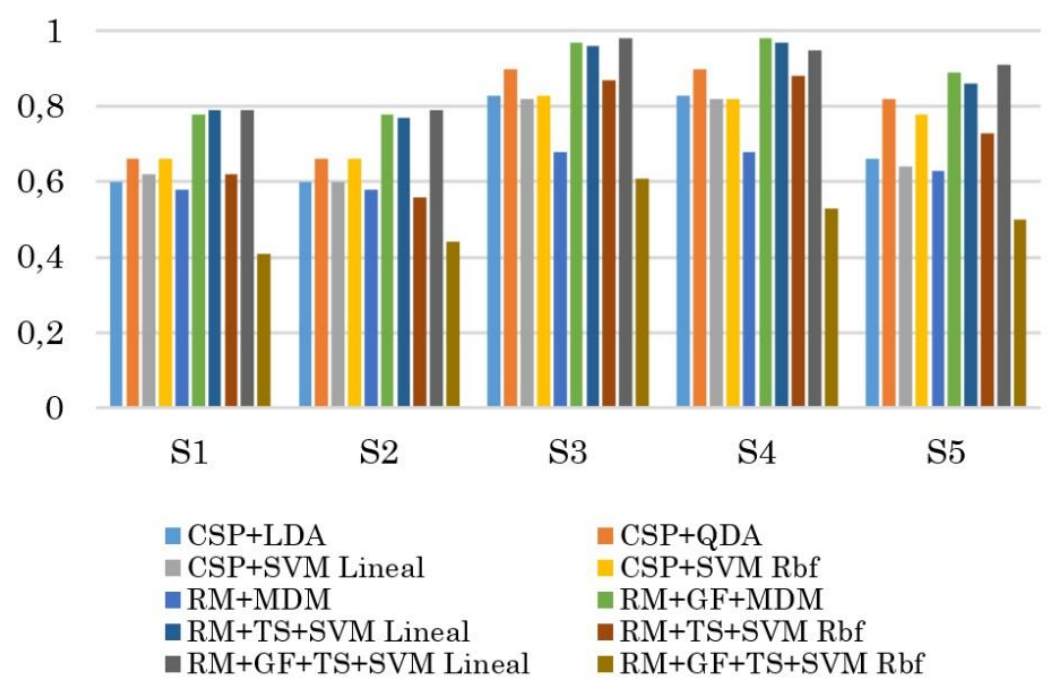

Fig. 7. AUC para cada miBCI. Fuente: autores. 
Evaluación del aporte de la covarianza de las señales electroencefalográficas a las interfaces cerebrocomputador de imaginación motora para pacientes con lesiones de médula espinal

Tabla 6. Valores de AUC para cada una de las diez miBCI por sujeto. Fuente: autores.

\begin{tabular}{lccccc}
\hline miBCI & S1 & S2 & S3 & S4 & S5 \\
\hline CSP+LDA & 0.60 & 0.60 & 0.83 & 0.83 & 0.66 \\
CSP+QDA & 0.66 & 0.66 & 0.90 & 0.90 & 0.82 \\
CSP+SVM Lineal & 0.62 & 0.60 & 0.82 & 0.82 & 0.64 \\
CSP+SVM Rbf & 0.66 & 0.66 & 0.83 & 0.82 & 0.78 \\
RM+MDM & 0.58 & 0.58 & 0.68 & 0.68 & 0.63 \\
RM+GF+MDM & 0.78 & 0.78 & 0.97 & $\mathbf{0 . 9 8}$ & 0.89 \\
RM+TS+SVM & $\mathbf{0 . 7 9}$ & 0.77 & 0.96 & 0.97 & 0.86 \\
Lineal & & & & & \\
RM+TS+SVM Rbf & 0.62 & 0.56 & 0.87 & 0.88 & 0.73 \\
RM+GF+TS+SVM & $\mathbf{0 . 7 9}$ & $\mathbf{0 . 8}$ & $\mathbf{0 . 9 8}$ & 0.95 & $\mathbf{0 . 9 1}$ \\
Lineal & & & & & \\
RM+GF+TS+SVM Rbf & 0.41 & 0.44 & 0.61 & 0.53 & 0.50 \\
\hline
\end{tabular}

Y, en segundo lugar, se observa que mientras las miBCI en los sujetos con lesiones cervicales (S1 y S2) presentan un desempeño regular, en los sujetos de control se logran miBCI con desempeños buenos (S5: $0.91 \quad-\quad$ miBCI: $\mathrm{RM}+\mathrm{GF}+\mathrm{TS}+\mathrm{SVM}$ Linear) y muy buenos (S3, S4: 0.98 - miBCI: RM+GF+TS+SVM Linear, $\quad \mathrm{RM}+\mathrm{GF}+\mathrm{MDM})$. El bajo desempeño de las arquitecturas propuesta con respecto a los sujetos de control puede deberse a la reorganización de la actividad cortical sensomotora [45] que tiene lugar en los pacientes que han sufrido lesiones en el sistema nervioso central como es el caso de los pacientes con lesiones cervicales, lo que indicaría que es necesario una reubicación de los electrodos sobre el cuero cabelludo de los sujetos con lesiones medulares para obtener bioseñales más discriminantes. Es posible que una sintonización de los hiperparámetros (los cuales se dejaron fijo para este estudio) mejore el desempeño particular las miBCI de Riemann que utilizan SVM de kernel gaussiano.

\section{CONCLUSIONES}

Se diseñaron e implementaron diez arquitecturas miBCI asociadas a imaginación de mano cerrada y abierta en tres sujetos de control y dos sujetos con lesiones medulares cervicales. Se analizó el aporte de la covarianza en la representación y discriminación de las señales EEG. Todas las miBCI tuvieron porcentajes de precisión por encima del nivel de azar.

Se encontró que, para ambos tipos de sujetos, las miBCI basadas en geometría de Riemann con clasificadores lineales mostraron mayor precisión que las miBCI basadas en CSP (tanto lineal como no lineal). Sin embargo, presentan mejor desempeño en los sujetos de control que en los sujetos con lesiones medulares.

Lo anterior sugiere que el desempeño de las miBCI que utilizan la información de covarianza de las señales EEG adquiridas con el Emotiv EPOC mejora cuando dicha información se utiliza para representar las señales EEG junto con modelos lineales de clasificación, que cuando se las utiliza con modelos no lineales o cuando la información de covarianza es utilizada en procesos de filtrado espacial de señales.

Los resultados obtenidos permiten entrever la posibilidad de desarrollar miBCI con equipos de bajo costo y algoritmos simples y robustos, utilizando la información de covarianza y los 
Evaluación del aporte de la covarianza de las señales electroencefalográficas a las interfaces cerebrocomputador de imaginación motora para pacientes con lesiones de médula espinal

elementos de la geometría de Riemann en clasificadores lineales.

Se espera, a futuro, que la sintonización de los híper-parámetros de las arquitecturas propuestas en los sujetos con lesiones medulares mejore el desempeño de las miBCI basadas en geometría de Riemann. También debe evaluarse la capacidad de generalización de estas miBCI con un número de épocas menores y en aplicaciones reales de generación de comandos a exomanos reales o virtuales, como apoyo a procesos de rehabilitación de miembros superiores en pacientes con LME.

\section{AGRADECIMIENTOS}

A Colciencias y a la Facultad de Ingeniería de la Universidad del Valle, en el marco de la convocatoria 727 del 2015, por el apoyo económico y administrativo.

\section{CONFLICTO DE INTERÉS}

Declaramos no tener ningún tipo de conflicto de intereses, ninguna relación personal, política, interés financiero ni académico que pueda influir en nuestro juicio.

\section{REFERENCIAS}

[1] A. S. Burns et al., "Type and Timing of Rehabilitation Following Acute and Subacute Spinal Cord Injury: A Systematic Review," Glob. Spine J., vol. 7, no. 3_suppl, p. 175 S-194S, Sep. 2017. https://doi.org/10.1177/2192568217703084

[2] J. van Tuijl, Y. Janssen-Potten, and $H$. Seelen, "Evaluation of upper extremity motor function tests in tetraplegics," Spinal Cord, vol. 40, no. 2, pp. 51-64, Feb. 2002. https://doi.org/10.1038/sj.sc.3101261

[3] Paralyzed Veterans of America Consortium for Spinal Cord Medicine, "Preservation of upper limb function following spinal cord injury: a clinical practice guideline for health-care professionals.," J. Spinal Cord Med., vol. 28, no. 5, pp. 434-470, Sep. 2005. https://doi.org/10.1080/10790268.2005.11753 844

[4] R. Rupp, S. C. Kleih, R. Leeb, J. del R. Millan, A. Kübler, and G. R. Müller-Putz, "Brain-Computer Interfaces and Assistive Technology," in Brain-Computer-Interfaces in their ethical, social and cultural contexts, vol. 12, Dordrecht: Springer Netherlands, 2014, pp. 7-38. https://doi.org/10.1007/978-94-017-8996-7_2

[5] G. Dornhege, J. del R. Millán, T. Hinterberger, D. J. McFarland, and K.-R. Müller, Toward Brain-Computer Interfacing, Cambridge: MIT Press, 2007.

[6] J. J. Shih, D. J. Krusienski, and J. R. Wolpaw, "Brain-Computer Interfaces in Medicine," Mayo Clin. Proc., vol. 87, no. 3, pp. 268-279, Mar. 2012.

https://doi.org/10.1016/j.mayocp.2011.12.008

[7] C. D. Ferrin Bolaños and H. Loaiza Correa, "Interfaz cerebro-computador multimodal para procesos de neurorrehabilitación de miembros superiores en pacientes con lesiones de médula espinal: una revisión," Rev. Ing. Biomédica, vol. 12, no. 24, pp. 3546, Dec. 2018.

https://doi.org/10.24050/19099762.n24.2018.1 222

[8] L. M. Alonso-Valerdi, R. A. Salido-Ruiz, and R. A. Ramirez-Mendoza, "Motor imagery based brain-computer interfaces: An emerging technology to rehabilitate motor deficits," Neuropsychologia, vol. 79 part B., pp. 354-363, Dec. 2015.

https://doi.org/10.1016/j.neuropsychologia.20 15.09.012

[9] P. H. Peckham et al., "Efficacy of an implanted neuroprosthesis for restoring hand grasp in tetraplegia: A multicenter study," Arch. Phys. Med. Rehabil., vol. 82, no. 10, pp. 1380-1388, Oct. 2001. https://doi.org/10.1053/apmr.2001.25910

[10] S. Mateo, F. Di Rienzo, V. Bergeron, A. Guillot, C. Collet, and G. Rode, "Motor imagery reinforces brain compensation of reach-to-grasp movement after cervical spinal cord injury," Front. Behav. Neurosci., vol. 9, pp. 1-12, Sep. 2015.

https://doi.org/10.3389/fnbeh.2015.00234

[11] M. Grangeon, P. Revol, A. Guillot, G. Rode, and C. Collet, "Could motor imagery be effective in upper limb rehabilitation of individuals with spinal cord injury? A case study," Spinal Cord, vol. 50, no. 10, pp. 766771, Oct. 2012.

https://doi.org/10.1038/sc.2012.41 
Evaluación del aporte de la covarianza de las señales electroencefalográficas a las interfaces cerebrocomputador de imaginación motora para pacientes con lesiones de médula espinal

[12] C. Vidaurre, C. Klauer, T. Schauer, A. Ramos-Murguialday, and K.-R. Müller, "EEG-based BCI for the linear control of an upper-limb neuroprosthesis," Med. Eng. Phys., vol. 38, no. 11, pp. 1195-1204, Nov. 2016.

https://doi.org/10.1016/j.medengphy.2016.06. 010

[13] J. Castillo, "Interfaz Cerebro-Computador Adaptativa Basada en Agentes Software para la Discriminación de Cuatro Tareas Mentales", Tesis Doctoral, Facultad de Ingeniería, Universidad del Valle, Cali, 2015. [En línea] Disponible en: http://hdl.handle.net/10893/10290

[14] A. Barachant, S. Bonnet, M. Congedo, and C. Jutten, "Classification of covariance matrices using a Riemannian-based kernel for BCI applications," Neurocomputing, vol. 112, pp. 172-178, Jul. 2013.

https://doi.org/10.1016/j.neucom.2012.12.039

[15] J. G. Proakis and D. G. Manolakis, Digital Signal Processing: Principles, Algorithms, and Applications, 3rd ed. New Jersey: Prentice-Hall, Inc., 1996.

[16] C. Bishop, Pattern Recognition and Machine Learning, 1st ed. New York: Springer-Verlag New York, 2006.

[17] C. M. Bishop, Neural networks for pattern recognition. New York: Oxford University Press, 1995.

[18] E. Giraldo-Suárez, J. I. Padilla-Buriticá, and C. G. Castellanos-Domínguez, "Dynamic inverse problem solution using a kalman filter smoother for neuronal activity estimation," TecnoLógicas, no. 27, p. 33-51, Dec. 2011.

https://doi.org/10.22430/22565337.3

[19] C. G. Lemus, "Análisis de reducción de ruido en señales eeg orientado al reconocimiento de patrones," Tecnológicas, no. 21, pp. 67-80, Dec. 2008.

https://doi.org/10.22430/22565337.248

[20] S. Mingxu, "A functional electrical stimulation (fes) control system for upper limb rehabilitation", PhD Thesis, School of Computing, Science and Engineering, University of Salford, Salford, 2014.

[En línea] Disponible en:

http://usir.salford.ac.uk/id/eprint/32854

[21] A. Barachant, S. Bonnet, M. Congedo, and C. Jutten, "Multiclass brain-computer interface classification by Riemannian geometry," IEEE Trans. Biomed. Eng., vol. 59, no. 4, pp. 920-928, Apr. 2012.

https://doi.org/10.1109/TBME.2011.2172210

[22] M. Tangermann et al., "Review of the BCI Competition IV," Front. Neurosci., vol. 6, no. 55, pp. 1-31, July. 2012.

https://doi.org/10.3389/fnins.2012.00055
[23] B. Blankertz, R. Tomioka, S. Lemm, M. Kawanabe, and K. Muller, "Optimizing Spatial filters for Robust EEG Single-Trial Analysis," IEEE Signal Process. Mag., vol. 25 , no. 1 , pp. 41-56, Dec. 2008.

https://doi.org/10.1109/MSP.2008.4408441

[24] F. Lotte and C. Guan, "Regularizing Common Spatial Patterns to Improve BCI Designs: Unified Theory and New Algorithms," IEEE Trans. Biomed. Eng., vol. 58, no. 2, pp. 355-362, Feb. 2011. https://doi.org/10.1109/TBME.2010.2082539

[25] S. Ge, R. Wang, and D. Yu, "Classification of Four-Class Motor Imagery Employing Single-Channel Electroencephalography," PLoS One, vol. 9, no. 6, p. e98019, Jun. 2014. https://doi.org/10.1371/journal.pone.0098019

[26] T. Hastie, R. Tibshirani, and J. Friedman, The Elements of Statistical Learning, 2nd ed. New York: Springer, 2017.

https://doi.org/10.1007/b94608

[27] S. Haykin, Neural Networks and Learning Machines, 3rd ed. Ontario: Pearson, Prentice Hall, 2008.

[28] A. Barachant, "Commande robuste d'un effecteur par une interface cerveau-machine EEG asynchrone", Tesis Doctoral, Electronique et système pour la santè, Université de Grenoble, Grenoble, 2006.

[En línea] Disponible en: https://tel.archives-ouvertes.fr/tel-01196752

[29] A. Barachant, S. Bonnet, M. Congedo, and C. Jutten, "Riemannian Geometry Applied to BCI Classification," in 9th International Conference Latent Variable Analysis and Signal Separation, France: Springer, 2010, pp. 629-636.

https://doi.org/10.1007/978-3-642-15995-4_78

[30] M. Congedo, A. Barachant, and A. Andreev, "A New Generation of Brain-Computer Interface Based on Riemannian Geometry," arXiv Prepr., p. 33, Oct. 2013.

[31] F. Yger, M. Berar, and F. Lotte, "Riemannian Approaches in Brain-Computer Interfaces: A Review," IEEE Trans. Neural Syst. Rehabil. Eng., vol. 25, no. 10, pp. 17531762, Oct. 2017.

https://doi.org/10.1109/TNSRE.2016.2627016

[32] K. Holewa and A. Nawrocka, "Emotiv EPOC neuroheadset in brain - computer interface," in Proceedings of the 2014 15th International Carpathian Control Conference (ICCC), Velke Karlovice, 2014, pp. 149-152. https://doi.org/10.1109/CarpathianCC.2014.6 843587

[33] J.-A. Martinez-Leon, J.-M. Cano-Izquierdo, and J. Ibarrola, "Are low cost Brain Computer Interface headsets ready for motor imagery applications?," Expert Syst. Appl., vol. 49, pp. 136-144, May. 2016. 
Evaluación del aporte de la covarianza de las señales electroencefalográficas a las interfaces cerebrocomputador de imaginación motora para pacientes con lesiones de médula espinal

https://doi.org/10.1016/j.eswa.2015.11.015

[34] N. A. Badcock et al., "Validation of the Emotiv EPOC EEG system for research quality auditory event-related potentials in children,” PeerJ, vol. 3, p. 17, Apr. 2015.

https://doi.org/10.7717/peerj.907

[35] J. Lu, D. J. McFarland, and J. R. Wolpaw, "Adaptive Laplacian filtering for sensorimotor rhythm-based brain-computer interfaces," J. Neural Eng., vol. 10, no. 1, p. 016002 , Dec. 2012.

https://doi.org/10.1088/17412560/10/1/016002

[36] F. Lotte, "A Tutorial on EEG Signalprocessing Techniques for Mental-state Recognition in Brain-Computer Interfaces," in Guide to Brain-Computer Music Interfacing, London: Springer London, 2014, pp. 133-161. https://doi.org/10.1007/978-1-4471-6584-2_7

[37] D. Wu, J.-T. King, C.-H. Chuang, C.-T. Lin, and T.-P. Jung, "Spatial Filtering for EEGBased Regression Problems in BrainComputer Interface (BCI)," IEEE Trans. Fuzzy Syst., vol. 26, no. 2, pp. 771-781, Apr. 2018.

https://doi.org/10.1109/TFUZZ.2017.2688423

[38] S. Yu, L.-C. Tranchevent, B. De Moor, and Y. Moreau, "Rayleigh Quotient-Type Problems in Machine Learning," in Kernel-based Data Fusion for Machine Learning: Methods and Applications in Bioinformatics and Text Mining, Heidelberg: Springer, 2011, pp. 2737.

https://doi.org/10.1007/978-3-642-19406-1_2

[39] D. P. Bertsekas, Nonlinear Programming, 3rd ed. Massachussets: Athena
Scientific, 2016.

[40] D. Watkins, "6. The Generalized Eigenvalue Problem," in The Matrix Eigenvalue Problem, 1st ed., Washington: Society for Industrial and Applied Mathematics, 2007, pp. 233263.

https://doi.org/10.1137/1.9780898717808.ch6

[41] T. Fushiki, "Estimation of prediction error by using K-fold cross-validation," Stat. Comput., vol. 21, no. 2, pp. 137-146, Apr. 2011. https://doi.org/10.1007/s11222-009-9153-8

[42] E. Combrisson and K. Jerbi, "Exceeding chance level by chance: The caveat of theoretical chance levels in brain signal classification and statistical assessment of decoding accuracy," J. Neurosci. Methods, vol. 250, pp. 126-136, Jul. 2015.

https://doi.org/10.1016/j.jneumeth.2015.01.01 0

[43] T. Fawcett, "An introduction to ROC analysis," Pattern Recognit. Lett., vol. 27, no. 8, pp. 861-874, Jun. 2006. https://doi.org/10.1016/j.patrec.2005.10.010

[44] A. Barachant, S. Bon, M. Congedo, and C. Jutten, "Common Spatial Pattern revisited by Riemannian geometry," in 2010 IEEE International Workshop on Multimedia Signal Processing, Saint Malo, 2010, pp. 472-476.

https://doi.org/10.1109/MMSP.2010.5662067

[45] K. A. Moxon, A. Oliviero, J. Aguilar, and G. Foffani, "Cortical reorganization after spinal cord injury: Always for good?," Neuroscience, vol. 283, pp. 78-94, Dec. 2014.

https://doi.org/10.1016/j.neuroscience.2014.06. 056 